\section{Depression and anxiety in} sterilised women in Iran

Sterilisation is an effective and convenient means of contraception and has become increasingly popular as a birth control technique throughout the world during the past 40 years. However some women who choose sterilisation may suffer a neurotic syndrome, which is manifested in the form of pain, depression and loss of libido. 1

We undertook a study designed to investigate depression, anxiety and post-operation regret rate in sterilised women referred to health centres in Tabriz, Iran in 2006. The study design was descriptive-analytical. The study participants comprised 300 women in the age range $25-45$ years, of whom 150 women were sterilised between 1 and 10 years ago and 150 were a control group of non-sterilised women who used condoms, withdrawal or safe period methods for contraception. The control group was selected by a cluster random sampling method. Fifteen health centres were selected as a cluster from 96 health centres located in Tabriz. Ten women were selected randomly from each health centre using health documents. Women were eligible for inclusion in the study if they were aged between 25 and 45 years at the time of sampling, and if they had no history of psychological disorders and no recent sorrowful events. There were no differences between the two groups as regards the number of children, income or demographic characteristics

The women were contacted by telephone at their last known address and were asked to complete questionnaires. Data collection was done using Zung's self-rating depression and anxiety scale in addition to questions about post-sterilisation regret. Data were collected from the subjects anonymously and analysed using SPSS (v. 11.5) statistics software. Analysis employed $t$-test, Chi-square test and descriptive statistics.

The comparison of the means for depression in the two groups was not significantly different $(p=0.96)$, however the mean of anxiety in the case group was remarkably greater than the control group $(p=0.03)$. Insufficient poststerilisation rest was a significant risk factor for depression and anxiety $(p=0.008$ and $p=0.02$. respectively). Requesting information about reversal after tubal sterilisation was $2.7 \%$ and the post-sterilisation regret rate was $6 \%$, which was significantly related to women's conflict with their husbands about the decision-making process prior to sterilisation $(p<0.001)$

The study findings as regards psychological disorders of sterilisation suggested that women undergoing sterilisation should ensure that they have a good rest after their operation in order to reduce the extent of psychological disorders. Unlike studies undertaken in other countries, ${ }^{2-4}$ women's age, parity, marriage duration and the timing of sterilisation was unrelated to the women's regret in our study. The earlier the sterilisation is carried out, the longer the woman's remaining period of fertile life and the greater the chances of changes in her marital status and/or the loss of a child, both circumstances that may lead to a change in the desired family size and expression of regret. In our study, probably one of the reasons why women's regret did not appear to be significantly related to young age of sterilisation was the infrequency of divorce or remarriage in our study population. Consistent with our study, Jamieson et al. reported that women who had substantial conflict with their husbands or partners prior to sterilisation were more than three times as likely to regret their decision and more than five times more likely to request a reversal than women who did not report such conflict. ${ }^{5}$

In our study, pre-sterilisation counselling was reported by $29.3 \%$ of subjects. With respect to personality and adaptability differences in facing the changes, pre-sterilisation counselling and post-sterilisation follow-up systems have an important role to play in women's psychological and psychosexual health promotion.

Noorizadeh Rogayeh, MSc

Midwifery Faculty, Marand University, Marand, Iran.E-mail:rahaparva@yahoo.com

Ivanbagha Reyhaneh, MSc

Nursing and Midwifery Faculty, Tabriz

University, Tabriz, Iran

Ranjbar Koochaksaraei Fatemeh, MD Psychiatry Department, Tabriz University of Medical Science, Tabriz, Iran

Pezeshki Mohammad Zakaria, MD

Social Medicine Department, Tabriz University of Medical Science, Tabriz, Iran

Bakhtari Aghdam Fateme, MS

Health and Nutrition Faculty, Tabriz University, Tabriz, Iran

\section{References}

Kaplan HI, Sadock BJ. Synopsis of Psychiatry: Behavioral Sciences, Clinical Psychiatry (8th edn) Publishers, 2000:55.

2 Hillis SD Marchbanks PA, Tylor LR, Peterson HB. Poststerilization regret. Obstet Gynecol 1999; 93: 889-895.

3 Schmidt JE, Hillis SD, Marchbanks PA, Jeng G, Peterson HB. Requesting information about and obtaining reversal after tubal sterilization. Fertil Steri

4 2000; 74: 892-898. Chuong CJ. Sequelae of postpartum sterilization. Arch

5 Jamieson DJ, Kaufman SC, Costello C, Hillis SD, Marchbanks PA, Peterson HB. A comparison of sterilization Obstet Gynecol 2002; 99. 1073-1079.

\section{Difficult insertion of IUS}

I would like to present a case of difficult insertion of an intrauterine system (IUS) due to the failure of the device to fully extrude from the applicator despite correct deployment.

The patient, a 34-year-old woman, gravida 3 para 0 , wished to have an IUS inserted following a medical termination of pregnancy. The termination had been quite an eventful procedure as the patient had profuse bleeding requiring dilatation and curettage and a blood transfusion. When she presented for the IUS fitting the bleeding had completely stopped. The IUS was inserted very easily as per the standard technique but on retrieving the inserter the device was still attached after what appeared to be a correct deployment. A second attempt with the same device yielded an identical result and it was not until a new device was used that the procedure could be completed successfully. Fearing operator failure, it was of some consolation to note that even when held in the hand, the device (which had an unusually large tail) did not leave the inserter after full deployment (Figure 1)

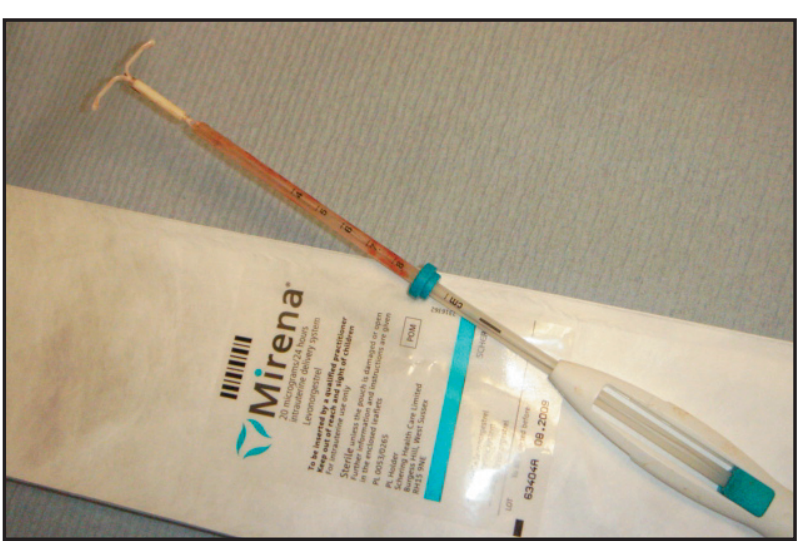

Figure 1 Photograph showing intrauterine system device still attached to inserte following unsuccessful deployment
Failure of intrauterine (IUD) or IUS deployment is likely to be an unreported event since the operator may blame themselves for not having (perhaps inadvertently) correctly deployed the device. However, it is extremely important to inspect all devices that fail in order to rule out manufacturing defects. The relative patency of our patient's cervical canal following the recent termination might have caused the faulty device to remain trapped in the inserter despite full and correct deployment. Conversely, a similarly defective device fitted in a woman with a tighter cervical os might have resulted in the device being released in the uterus but in an abnormally low position after having been dragged by the introducer on its withdrawal. In such a situation the operator would be totally unaware of the device malfunction, and the abnormally low positioning could lead to device expulsion.

The present case occurred with an IUS but it is not unreasonable to imagine that a similar mechanism could apply to different IUDs such as the TT380 Slimline ${ }^{\circledR}{ }_{1,2}$ It is thus important to collect for inspection any devices that fail to deploy correctly since this might shed some light on the reason(s) for expulsion and might perhaps lead to better quality control procedures for the device itself.

Paola Albertazzi, MD, FRCOG,

Staff Grade, Abacus, Contraception and Reproductive Health Services, Liverpool, UK.

E-mail: albertazzi@doctors.org.uk

\section{References}

Hawkins F, Callander N. Increase in IUD expulsions Letter]. J Fam Plann Reprod Health Care 2006; 32

Yadava RP. Increase in IUD expulsions [Letter]. J Fam Plann Reprod Health Care 2007; 33: 66-67.

\section{Reasons for IUD/IUS removal}

Intrauterine devices (IUDs) and the intrauterine system (IUS) are more cost effective than oral contraception. 1 Evidence from our clinics suggests that devices were being removed earlier than recommended. We therefore reviewed client contacts during 2005 in two clinics to inquire about the reasons for device removal. Table 1 shows the duration of use of IUDs at clinic attendance. The lower section of the table shows the duration of use at removal.

Of 40 devices removed, nine $(22.5 \%)$ were 'time expired' (i.e. the device was beyond its recommended duration of use). Seven $(17.5 \%)$ were removed due to bleeding problems, six $(15 \%)$ were extruded and five $(12.5 \%)$ were removed to facilitate pregnancy.

Almost half $(45 \%)$ the removals were because the devices had served their purpose. These were 'time expired' (i.e. partner had vasectomy, menopause, etc.). The remaining 55\% of devices were removed for complications or other reasons. The commonest reason for removals was bleeding (17.5\% of clients). 